\title{
Distribuição do fósforo inorgânico em sistemas de manejo de solo(1)
}

\author{
Danilo dos Santos Rheinheimer(2) e Ibanor Anghinoni(3)
}

\begin{abstract}
Resumo - A intensidade das reações dos fertilizantes fosfatados no solo deve variar em razão dos diferentes sistemas de manejo do solo. Este trabalho teve o objetivo de avaliar as modificações nas frações de $\mathrm{P}$ inorgânico provocadas por diferentes preparos do solo e sucessões de cultura. Coletaram-se, em maio de 1997, amostras de solo (Latossolo Vermelho distroférrico típico, Latossolo Vermelho distrófico típico e Argissolo Vermelho distrófico típico), em três camadas (0-2,5, 2,5-7,5 e 7,5-17,5 cm), de quatro experimentos instalados a partir de 1979, envolvendo os sistemas plantio direto e cultivo convencional com diferentes sucessões de cultura. Foram determinados o $\mathrm{P}$ total e seis frações de $\mathrm{P}$ inorgânico, em ordem decrescente de labilidade. O conteúdo médio de $\mathrm{P}$ total foi bem superior ao do seu estado natural, e no sistema plantio direto ocorreu um grande acúmulo na camada superficial. Neste sistema, recuperaram-se maiores teores de $\mathrm{P}$ inorgânico lábil (resina e $\mathrm{NaHCO}_{3}$ $\left.0,5 \mathrm{~mol} \mathrm{~L}^{-1}\right)$ e também não-lábil ligado ao $\mathrm{Ca}\left(\mathrm{HCl} 1,0 \mathrm{~mol} \mathrm{~L}^{-1}\right)$ na camada superficial. As maiores concentrações de $\mathrm{P}$ inorgânico foram extraídas pelo $\mathrm{NaOH} 0,1 \mathrm{~mol} \mathrm{~L}^{-1}$, ditas moderadamente lábeis. As sucessões de cultura tiveram pouca influência nas frações de P inorgânico.
\end{abstract}

Termos para indexação: preparo do solo, adubação fosfatada, culturas, absorção de nutrientes.

\section{Distribution of inorganic phosphorus fraction in soil management systems}

\begin{abstract}
The intensity of the reactions of the phosphate fertilizer in the soil should vary in function of the different management systems. The study had the objective of evaluating the modifications in the inorganic fractions of P as affected by soil tillage and crop sequence. In May 1997, three soil layers of four long-term field experiments, involving no-tillage and conventional tillage with different crop sequences, were sampled. The total $\mathrm{P}$ and six fractions of inorganic $\mathrm{P}$ were evaluated. The medium content of total $\mathrm{P}$ was much higher than the native $\mathrm{P}$. Total $\mathrm{P}$ increased in the upper soil layer under notillage. Available (resin and $\left.\mathrm{NaHCO}_{3} 0.5 \mathrm{~mol} \mathrm{~L}^{-1}\right)$ and nonlabile inorganic $\mathrm{P}\left(\mathrm{HCl} 1.0 \mathrm{~mol} \mathrm{~L}^{-1}\right)$ were higher in the surface layer under no-tillage than in conventional tillage. The highest concentrations of inorganic $\mathrm{P}$ were moderately labile $\left(\mathrm{NaOH} 0.1 \mathrm{~mol} \mathrm{~L}^{-1}\right)$. The crop sequences did not influence the fractions of inorganic $\mathrm{P}$.
\end{abstract}

Index terms: tillage, phosphate fertilizers, crops, nutrient uptake.

\section{Introdução}

O P contido no material de origem do solo encontra-se totalmente na forma mineral, com predomínio dos fosfatos de Ca. Através da intemperização, este

\footnotetext{
(1) Aceito para publicação em 12 de abril de 2000. Trabalho realizado com apoio financeiro da FINEP/FAPERGS.

(2) Universidade Federal de Santa Maria (UFSM), Centro de Ciências Rurais (CCR), Dep. de Solos, Caixa Postal 221, CEP 97105-900 Santa Maria, RS. E-mail: danilo@ ccr.ufsm.br

(3) Universidade Federal do Rio Grande do Sul (UFRGS), Faculdade de Agronomia, Dep. de Solos, Caixa Postal 776, CEP 91540-000 Porto Alegre, RS. E-mail: ibanghi@ vortex.ufrgs.br
}

nutriente é liberado para a solução do solo. Concomitantemente, ocorrem perdas de bases, sílica e carbonatos, e aumento na atividade de elementos como o $\mathrm{Al}$ e o Fe, com a conseqüente transformação dos minerais primários em argilas 2:1 e estas em 1:1 e óxidos. A partir daí, formam-se minerais fosfatados mais estáveis termodinamicamente; parte do $\mathrm{P}$ é adsorvido à superfície de minerais secundários, e parte é absorvida e incorporada pela biomassa e matéria orgânica do solo (Walker \& Syers, 1976; Smeck, 1985). De acordo com este paradigma, ambos os processos, geoquímico e biológico, transformam os fosfatos naturais do solo em formas orgânicas e inorgânicas estáveis, com concomitante transferên- 
cia das formas mais solúveis para os ambientes aquáticos. Em solos altamente intemperizados, predominam as formas inorgânicas ligadas à fração mineral com alta energia e as formas orgânicas estabilizadas física e quimicamente. Isto sugere que os teores de $\mathrm{P}$ total e a distribuição nas diferentes frações dependem do grau de intemperização, das características químicas e físicas do solo, da atividade biológica e da vegetação predominante, entre outras (Magid, 1993; Cross \& Schlesinger, 1995).

O conteúdo de $\mathrm{P}$ total dos solos em seus ambientes naturais, no Rio Grande do Sul, RS, varia de menos de 100 até mais de $1.000 \mathrm{mg} \mathrm{dm}^{-3}$, dependendo do material de origem e do grau de evolução genética. Solos derivados de basalto são mais ricos em $\mathrm{P}$ do que os derivados de rochas sedimentares (Machado et al., 1993). A distribuição das formas inorgânicas de $\mathrm{P}$ varia de acordo com a mineralogia do solo. Estes autores encontraram mais baixo porcentual de formas ativas ou lábeis nos solos da região do Planalto do que nos da região sul do RS, em virtude dos maiores teores de argila e óxidos de ferro. $\mathrm{O}$ uso dos solos para as atividades agrícolas aumentou os teores de $\mathrm{P}$ total da camada arável, pois as quantidades desse nutriente adicionadas através dos fertilizantes fosfatados, nas últimas décadas, superaram as quantidades exportadas pelas colheitas e pela erosão, alterando a magnitude das frações inorgânicas (Ball-Coelho et al., 1993; Beck \& Sanchez, 1994; Araújo \& Salcedo, 1997).

No sistema plantio direto (SPD), os fertilizantes são aplicados na superfície do solo sem a posterior incorporação, o que tem aumentado grandemente os teores de P total da camada superficial. No entanto, o aumento nas formas orgânicas não acompanha o rápido incremento no $\mathrm{P}$ total (Selles et al., 1997; Rheinheimer et al., 1998). Também, tem-se observado que podem ocorrer aumentos nos teores de $\mathrm{Ce}$ $\mathrm{N}$ total sem alterar os teores de $\mathrm{P}$ orgânico (Kingery et al., 1996). Isto indica que a ciclagem do $P$ é menos eficiente do que a do $\mathrm{N}$ e do $\mathrm{C}$, pois sofre reações de adsorção, e é mineralizado preferencialmente, o que resulta em acúmulo pronunciado de $\mathrm{P}$ inorgânico na camada superficial. Kunishi et al. (1982), também, constataram que o $\mathrm{P}$ adicionado na superfície permaneceu como fosfato de $\mathrm{Ca}$, tornando-se menos adsorvido pelos colóides do solo sob SPD por três anos.
As plantas apresentam mecanismos que auxiliam na aquisição de $P$, seja pela modificação na solubilização-adsorção, seja na difusão do nutriente no solo. Estas estratégias se manifestam pela maior relação raiz/parte aérea e pelo aumento da superfície radicular, pela maior taxa de absorção por unidade de raiz, pelo aumento da exsudação radicular de fosfatases e outros compostos orgânicos, e pela alteração do grau de micotrofismo (Lajtha \& Harrison, 1995). Deste modo, os resíduos vegetais apresentam composição química bastante variável, tanto quantitativa como qualitativamente, em relação ao $\mathrm{P}$, cuja disponibilidade a curto prazo pode ser alterada. $\mathrm{O}$ efeito da qualidade dos resíduos vegetais na distribuição das frações de P inorgânico do solo é pouco conhecido na literatura internacional (Magid, 1993), e desconhecido em relação aos solos da Região Sul do Brasil.

O presente trabalho teve por objetivo avaliar a distribuição das frações de P inorgânico em solos com diferentes sistemas de preparo e sucessões de cultura.

\section{Material e Métodos}

Usaram-se amostras de solos de quatro experimentos de diferentes locais do Estado do Rio Grande do Sul (Tabela 1).

O primeiro experimento foi instalado em 1979, em Latossolo Vermelho distroférrico típico, muito argiloso, substrato basalto (LVdf - Rhodic Hapludox) no Centro de Atividades Agrícolas e Florestais da Cooperativa Tritícola de Santo Ângelo, em Santo Ângelo, RS, cuja área tinha sido cultivada com trigo (Triticum aestivum L.) / soja (Glyxine max (L.) Merrill) por 15 anos e apresentava avançado processo de degradação física (baixa taxa de infiltração, compactação na camada $15-20 \mathrm{~cm}$, solo desestruturado, erosão laminar forte). Atualmente, os tratamentos constam de três rotações de culturas e três leguminosas antecedendo o milho (Zea mays L.) no sistema plantio direto (SPD) e as sucessões trigo/soja e aveia (Avena strigosa Schreb.) / milho sob SPD, cultivo convencional (SCC) e cultivo mínimo. Aplicaram-se, em média (a variação é dependente da safra e da cultura utilizada), $23 \mathrm{~kg} \mathrm{ha}^{-1}$ de $\mathrm{P}$ e foram exportados pelos grãos aproximadamente (teor médio nos grãos e produtividade média dos tratamentos) $12 \mathrm{~kg} \mathrm{ha}^{-1}$ de $\mathrm{P}$, em cada cultivo comercial. Neste trabalho, utilizaram-se amostras das parcelas sem N do SPD com as sucessões trigo/soja e aveia/milho e uma das rotações de culturas e do SCC das 
duas sucessões de culturas. Na sucessão aveia/milho, haviam sido cultivados trigo/soja até a safra 85/86. Na rotação de culturas, foram cultivados trigo, soja, tremoço (Lupinus angustifolium L.), milho, sorgo (Sorghum bicolor L.) e aveia-preta + trevo (Trifolium repens L.).

O segundo experimento foi instalado em 1983, em Latossolo Vermelho distrófico típico, argiloso, substrato basalto (LVd - Rhodic Hapludox), na Embrapa-Centro Nacional de Pesquisa de Trigo, em Passo Fundo, RS. Os tratamentos envolveram os mesmos três métodos de preparo do solo. O experimento foi composto por três blocos, nos quais foram introduzidas as culturas de ervilhaca (Vicia sativa L.), milho, sorgo, aveia-preta, soja e cevada (Hordeum vulgare L.), variáveis no tempo. Aplicou-se, em média, um total de $17,5 \mathrm{~kg} \mathrm{ha}^{-1}$ de $\mathrm{P}$ e foram exportados pelos grãos aproximadamente $14 \mathrm{~kg} \mathrm{ha}^{-1}$ de $\mathrm{P}$ em cada cultivo comercial. Neste estudo, coletaram-se amostras de solo do SPD e SCC logo após a colheita das culturas de soja e sorgo.

O terceiro experimento foi instalado em 1985, em Argissolo Vermelho distrófico típico (PVd - Rhodic Paleudults), franco argiloso-arenoso, degradado fisicamente, na Estação Experimental Agronômica da Universidade Federal do Rio Grande do Sul, em Eldorado do Sul, RS. Os tratamentos consistiram dos três métodos de preparo do solo, três sucessões de cultura (aveia/milho, aveia+ervilhaca/milho e aveia+ervilhaca/milho+caupi (Vigna unguiculata subsp. unguiculata (L.) Walp.)) e duas doses de N. Aplicou-se, em média, um total de $20 \mathrm{~kg} \mathrm{ha}^{-1}$ de $\mathrm{P}$, e foram exportados pelos grãos aproximadamente $15 \mathrm{~kg} \mathrm{ha}^{-1}$ de $\mathrm{P}$ em cada cultivo comercial. Neste trabalho, usaram-se amostras das parcelas sem $\mathrm{N}$ do SPD e SCC nas sucessões aveia/milho e aveia+ervilhaca/milho+caupi.

Tabela 1. Características químicas e granulométricas dos $\operatorname{solos}^{(1)}$

\begin{tabular}{lccc}
\hline Característica & $\begin{array}{c}\text { LVdf } \\
\text { Rhodic } \\
\text { Hapludox }\end{array}$ & $\begin{array}{c}\text { LVd } \\
\text { Rhodic } \\
\text { Hapludox }\end{array}$ & $\begin{array}{c}\text { PVd } \\
\text { Rhodic } \\
\text { Paleudult }\end{array}$ \\
\hline Argila $\left(\mathrm{g} \mathrm{kg}^{-1}\right)$ & 680 & 530 & 220 \\
$\mathrm{Fe}_{\mathrm{d}}\left(\mathrm{g} \mathrm{kg}^{-1}\right)$ & 246 & 56 & 36 \\
Caulinita $\left(\mathrm{g} \mathrm{kg}^{-1}\right)$ & 710 & 710 & 720 \\
$\mathrm{Fe}_{\mathrm{d}}\left(\mathrm{g} \mathrm{kg}^{-1}\right)$ & 274 & 160 & 109 \\
Carbono $\left(\mathrm{g} \mathrm{kg}^{-1}\right)$ & 27 & 22 & 21 \\
Fósforo $\left(\mathrm{mg} \mathrm{kg}^{-1}\right)$ & 2 & 4 & 7 \\
\hline
\end{tabular}

(1) Resultado médio das três camadas amostradas $(0-2,5,2,5-7,5$ e 7,5-17,5 cm); argila determinada pelo método do densímetro; ferro extraído por ditionito-citrato-bicarbonato de sódio; carbono determinado por digestão úmida; fósforo extraído por Mehlich 1; LVdf: Latossolo Vermelho distroférrico típico; LVd: Latossolo Vermelho distrófico típico; PVd: Argissolo Vermelho distrófico típico.
O quarto experimento foi instalado em 1983, em área adjacente ao experimento acima descrito. Os tratamentos utilizados inicialmente foram dois níveis de compactação do solo (sem e com descompactação por subsolagem), dez sistemas de culturas, e duas doses de N, conduzido exclusivamente sob SPD. Foram aplicados, em média, $20 \mathrm{~kg} \mathrm{ha}^{-1}$ de $\mathrm{P}$, e foram exportados pelos grãos aproximadamente $15 \mathrm{~kg} \mathrm{ha}^{-1}$ de $\mathrm{P}$ em cada cultivo comercial. Neste trabalho, usaram-se amostras das parcelas sem $\mathrm{N}$ e solo descompactado, nos sistemas aveia/milho, aveia + ervilhaca/milho + caupi e guandu (Cajanus cajan (L.) Millps) + milho.

Em maio de 1997, logo após o preparo do solo, exceto no SPD, coletaram-se amostras de solo, em duas repetições de cada tratamento, nas camadas de 0-2,5, 2,5-7,5 e $7,5-17,5 \mathrm{~cm}$. Cada amostra foi composta pela homogeneização de duas subamostras oriundas de trincheiras de $50 \times 10 \mathrm{~cm}$, coletadas perpendicularmente às linhas de semeadura. $\mathrm{O}$ solo foi secado ao ar e peneirado em malha de $1 \mathrm{~mm}$.

$\mathrm{O} P$ total foi determinado após extração com $\mathrm{H}_{2} \mathrm{SO}_{4}+$ $\mathrm{H}_{2} \mathrm{O}_{2}$ a quente. No fracionamento do $\mathrm{P}$ total, as amostras de $1,50 \mathrm{~g}$ de solo foram submetidas a diferentes extratores, num esquema seqüencial (Hedley et al., 1982). Inicialmente, foi analisado o P inorgânico extraído por resinas trocadoras de ânions em membranas, AR 103 QDP 434, saturadas com $\mathrm{NaHCO}_{3}$ 0,5 mol L-1 (Pi-resina); em seguida, extraiu-se o P inorgânico com $\mathrm{NaHCO}_{3} 0,5$ mol L-1 $\left(\mathrm{Pi}-\mathrm{NaHCO}_{3}\right)$, quimiossorvido com baixa energia; com $\mathrm{NaOH}$ 0,1 mol L-1 (Pi-NaOH 0,1), quimiossorvido com média energia; com $\mathrm{HCl} 1,0 \mathrm{~mol} \mathrm{~L}^{-1}(\mathrm{Pi}-\mathrm{HCl})$, fosfatos de $\mathrm{Ca}$ e com $\mathrm{NaOH} 0,5 \mathrm{~mol} \mathrm{~L}^{-1}(\mathrm{Pi}-\mathrm{NaOH} 0,5)$, quimiossorvido com alta energia. $\mathrm{O}$ resíduo de solo foi digerido com $\mathrm{H}_{2} \mathrm{SO}_{4}+\mathrm{H}_{2} \mathrm{O}_{2}+\mathrm{MgCl}_{2}$ saturado (P-residual) (Brookes \& Powlson, 1982). O P inorgânico dos extratos alcalinos foi determinado usando-se o procedimento descrito por Dick \& Tabatabai (1977). Em amostras separadas determinou-se o P extraído por Mehlich I (Mehlich, 1953).

\section{Resultados e Discussão}

O conteúdo de $\mathrm{P}$ total no LVdf é bastante elevado, devido à riqueza do material de origem (basalto), que, aliado a adições em quantidades superiores às exportadas pelos grãos das culturas, apresentaram teores próximos a $1.000 \mathrm{mg} \mathrm{dm}^{-3}$ (Tabela 2). Na camada $0-2,5 \mathrm{~cm}$, o solo sob SPD contém maior teor de P total em relação ao solo sob SCC; o inverso foi observado na camada 7,5-17,5 cm. Isso ocorre pela não-incorporação dos fertilizantes adicionados na superfície, às menores perdas por erosão, e, também, 
pela reciclagem proporcionada pelas plantas, as quais absorvem o P disponível de camadas mais profundas, deixando-o na superfície, quando da decomposição dos seus resíduos. A média ponderada com as espessuras das camadas não diferiu entre os métodos de preparo do solo e entre as sucessões de cultura.

No LVdf, o teor de Pi-resina extraída do solo sob SCC foi menor na camada $0-2,5 \mathrm{~cm}$ em relação aos das demais profundidades, comportamento, este, contrário ao constatado no SPD (Tabela 2). Neste, o $\mathrm{P}$ adicionado como fertilizante, na linha de semeadura, permaneceu na camada superficial e em formas mais disponíveis, cujo valor foi mais do que o dobro do valor do solo sob SCC (63 e $30 \mathrm{mg} \mathrm{dm}^{-3}$ ). O solo da região próxima à dissolução do fertilizante é saturado pelos produtos da reação solo-fertilizante, diminuindo a energia de ligação do fosfato com os colóides do solo. Também, neste sistema, os teores de matéria orgânica são mais elevados, cujas subs- tâncias húmicas, ou mesmo ácidos orgânicos de baixa massa molecular, podem auxiliar na diminuição dos sítios de adsorção e da energia de ligação fosfatosolo. Independentemente do método de preparo do solo, o maior teor de Pi-resina foi constatado na sucessão aveia/milho, e o menor, na rotação (Tabela 2). $\mathrm{O}$ bicarbonato de sódio extraiu maior teor de $\mathrm{P}$ inorgânico na camada superficial do SPD $\left(44 \mathrm{mg} \mathrm{dm}^{-3}\right)$ do que do SCC $\left(27 \mathrm{mg} \mathrm{dm}^{-3}\right)$, embora grande parte do $\mathrm{P}$ fracamente adsorvido já tivesse sido retirada pela resina, o bicarbonato de sódio extraiu uma considerável quantidade deste nutriente, o qual é, também, considerado disponível (Schmidt et al., 1996; Guo \& Yost, 1998).

Os teores de $\mathrm{P}$ inorgânico extraídos pelo $\mathrm{NaOH}$ $0,1 \mathrm{~mol} \mathrm{~L}^{-1}$ no LVdf não diferiram, devido aos métodos de preparo do solo e às sucessões de cultura. Os valores, médias dos dois métodos de preparo, e as três sucessões de cultura, foram de 134, 154

Tabela 2. Fósforo total e frações de fósforo inorgânico (Pi) do Latossolo Vermelho distroférrico típico, em razão de métodos de preparo do solo, sucessões de cultura e camadas amostradas ${ }^{(1)}$.

\begin{tabular}{|c|c|c|c|c|c|c|c|c|}
\hline \multirow{2}{*}{$\begin{array}{l}\text { Fração de } \\
\text { fósforo }\end{array}$} & \multirow{2}{*}{$\begin{array}{c}\text { Preparo } \\
\text { do solo }\end{array}$} & \multicolumn{3}{|c|}{ Camadas amostradas $(\mathrm{cm})^{(2)}$} & \multicolumn{3}{|c|}{ Sucessões de cultura $^{(3)}$} & \multirow[t]{2}{*}{ C.V } \\
\hline & & $0-2,5$ & $2,5-7,5$ & $7,5-17,5$ & $\mathrm{~A} / \mathrm{M}$ & $\mathrm{T} / \mathrm{S}$ & Rotação & \\
\hline & & \multicolumn{6}{|c|}{ - } & $(\%)$ \\
\hline \multirow[t]{2}{*}{ P total } & SPD & $1.151 \mathrm{aA}$ & $1.083 \mathrm{abA}$ & 955bB & & & & \\
\hline & SCC & $976 \mathrm{bB}$ & $1.154 \mathrm{aA}$ & $1.085 \mathrm{aA}$ & $1.084 \mathrm{a}$ & $1.079 \mathrm{a}$ & $1.007 \mathrm{a}$ & 8,2 \\
\hline \multirow{2}{*}{ Pi-resina } & SPD & $63 \mathrm{aA}$ & $49 \mathrm{bA}$ & $25 \mathrm{cB}$ & & & & \\
\hline & $\mathrm{SCC}$ & $30 \mathrm{bB}$ & $48 \mathrm{aA}$ & $49 \mathrm{aA}$ & $53 a$ & $42 b$ & $34 c$ & 16,1 \\
\hline \multirow{2}{*}{$\begin{array}{l}\mathrm{Pi}-\mathrm{NaHCO}_{3} \\
0,5 \mathrm{~mol} \mathrm{~L}^{-1}\end{array}$} & SPD & $44 \mathrm{aA}$ & $35 \mathrm{bA}$ & $32 \mathrm{bA}$ & & & & \\
\hline & SCC & $27 \mathrm{aB}$ & $33 \mathrm{aA}$ & $29 \mathrm{aA}$ & $37 \mathrm{a}$ & $35 \mathrm{a}$ & $38 \mathrm{a}$ & 21,5 \\
\hline \multirow{2}{*}{$\begin{array}{l}\mathrm{Pi}-\mathrm{NaOH} \\
0,1 \mathrm{~mol} \mathrm{~L}^{-1}\end{array}$} & SPD & $138 \mathrm{aA}$ & $147 \mathrm{aA}$ & $129 \mathrm{aA}$ & & & & \\
\hline & SCC & $130 \mathrm{bA}$ & $161 \mathrm{aA}$ & $135 \mathrm{bA}$ & $148 \mathrm{a}$ & $134 \mathrm{a}$ & $134 a$ & 12,2 \\
\hline \multirow{2}{*}{$\begin{array}{l}\mathrm{Pi}-\mathrm{HCl} \\
1,0 \mathrm{~mol} \mathrm{~L}^{-1}\end{array}$} & SPD & $43 \mathrm{aA}$ & $29 \mathrm{bA}$ & $14 \mathrm{cA}$ & & & & \\
\hline & SCC & $23 \mathrm{abB}$ & $27 \mathrm{aA}$ & $16 \mathrm{bA}$ & $23 a$ & $26 a$ & $30 \mathrm{a}$ & 21,2 \\
\hline \multirow{2}{*}{$\begin{array}{l}\mathrm{Pi}-\mathrm{NaOH} \\
0,5 \mathrm{~mol} \mathrm{~L}^{-1}\end{array}$} & SPD & $165 \mathrm{aA}$ & $158 \mathrm{aA}$ & $126 \mathrm{bA}$ & & & & \\
\hline & SCC & $140 \mathrm{bB}$ & $164 \mathrm{aA}$ & $142 \mathrm{bA}$ & $145 \mathrm{a}$ & $154 \mathrm{a}$ & $150 \mathrm{a}$ & 10,1 \\
\hline \multirow[t]{2}{*}{ P-residual } & SPD & $217 \mathrm{bA}$ & $287 \mathrm{aB}$ & $290 \mathrm{aB}$ & & & & \\
\hline & SCC & $222 \mathrm{bA}$ & $339 \mathrm{aA}$ & $335 \mathrm{aA}$ & $281 \mathrm{a}$ & $288 \mathrm{a}$ & $275 a$ & 14,8 \\
\hline \multirow[t]{2}{*}{ P-Mehlich } & SPD & $110 \mathrm{aA}$ & $60 \mathrm{bA}$ & $34 \mathrm{cA}$ & & & & \\
\hline & SCC & $52 \mathrm{abB}$ & $61 \mathrm{aA}$ & $43 \mathrm{bA}$ & $58 \mathrm{a}$ & $68 \mathrm{a}$ & $62 \mathrm{a}$ & 23,2 \\
\hline
\end{tabular}


e $132 \mathrm{mg} \mathrm{dm}^{-3}$ nas camadas $0-2,5,2,5-7,5 \mathrm{e}$ 7,5-17,5 cm, respectivamente, representando 18, 20 e $19 \%$ do Pi total. Similarmente, o $\mathrm{NaOH} 0,5 \mathrm{~mol} \mathrm{~L}^{-1}$ extraiu 21, 21 e $19 \%$ do Pi total nas referidas camadas (Tabela 2). Deste modo, aproximadamente $40 \%$ do Pi total foi extraído nas extrações sucessivas com $\mathrm{NaOH}$, por causa da mineralogia do solo, representando o P coordenado ao $\mathrm{Fe}$ e $\mathrm{Al}$ dos óxidos e na superfície da caulinita, com moderada energia. Estas frações, especialmente a primeira $(\mathrm{NaOH}$ $0,1 \mathrm{~mol} \mathrm{~L}^{-1}$ ), atuam como dreno de $\mathrm{P}$ em solos fertilizados, ou como uma fonte a curto e médio prazo à biomassa. Sua ação como tamponante pode manter os níveis de $\mathrm{P}$ suficientemente altos para o suprimento das plantas. Assim, o P inorgânico extraído com $\mathrm{NaOH}$ pode ser considerado moderadamente lábil (Ball-Coelho et al., 1993; Beck \& Sanchez, 1994; Schmidt et al., 1996; Araújo \& Salcedo, 1997; Guo $\&$ Yost, 1998).

A fração de $\mathrm{P}$ extraída pelo $\mathrm{HCl} 1,0 \mathrm{~mol} \mathrm{~L}^{-1}$ no LVdf foi muito superior na camada superficial do SPD (43 $\mathrm{mg} \mathrm{dm}^{-3}$ ), em comparação com a do SCC $\left(23 \mathrm{mg} \mathrm{dm}^{-3}\right)$. Como os fosfatos de Ca são, praticamente, ausentes neste solo (Machado et al., 1993), essa fração deve ser de fosfatos de Ca secundários (Magid, 1993). Considerando-se que os sítios de adsorção vão sendo paulatinamente saturados, é de se esperar que, a longo prazo, a concentração de $\mathrm{P}$ na solução do solo na região de influência da difusão dos produtos de reação solo-fertilizante seja alta o suficiente para a neoformação de fosfatos de mais baixa solubilidade. Kunishi et al. (1982) já tinham observado que os fertilizantes fosfatados aplicados na superfície do solo sob SPD permaneciam por maior tempo como fosfatos de $\mathrm{Ca}$, devido à diminuição da adsorção com os colóides minerais. Essa fração tem sido considerada não-lábil (Ball-Coelho et al., 1993; Schmidt et al., 1996; Selles et al., 1997; Guo \& Yost, 1998).

A fração de P-residual no LVdf foi maior no SCC do que no SPD, nas duas camadas mais profundas, e estas foram superiores à camada $0-2,5 \mathrm{~cm}$. As sucessões de cultura não alteraram os teores de P-residual (Tabela 2). Esta fração é constituída por formas insolúveis de $\mathrm{P}$ inorgânico, e mais estáveis de $\mathrm{P}$ orgânico, sendo considerada recalcitrante. Os altos teores de P-residual estão de acordo com a alta densida- de de sítios de adsorção de fosfatos, uma vez que esse solo apresenta mais de $70 \%$ de argila, com predominância absoluta da caulinita e óxidos de $\mathrm{Fe}$ (Bayer, 1996).

O teor de P extraído pelo método de Mehlich I no LVdf foi maior na camada superficial do SPD $\left(110 \mathrm{mg} \mathrm{dm}^{-3}\right)$ do que o do SCC (52 $\left.\mathrm{mg} \mathrm{dm}^{-3}\right)$, e não foi afetado pelas sucessões de cultura. Os valores são muitas vezes superiores ao nível crítico estabelecido pela Comissão de Fertilidade do Solo (1995), que é de $6 \mathrm{mg} \mathrm{dm}^{-3}$.

O conteúdo de $\mathrm{P}$ total no $\mathrm{LVd}$ também é elevado, pois apresenta o basalto como material de origem (Tabela 3). O teor de P total foi semelhante nas culturas precedentes. No SCC ocorreu distribuição uniforme no perfil amostrado $(0-17,5 \mathrm{~cm})$, pela mobilização do solo para a implantação das culturas, cuja média ponderada foi de $754 \mathrm{mg} \mathrm{dm}^{-3}$. No $\mathrm{SPD}$, os valores foram de $1.085,1.068 \mathrm{e} 897 \mathrm{mg} \mathrm{dm}^{-3}$ nas camadas 0-2,5, 2,5-7,5 e 7,5-17,5 cm, respectivamente; nas duas primeiras camadas as médias foram superiores às encontradas no $\mathrm{SCC}(\mathrm{p}<0,05)$.

No LVd a quantidade de Pi-resina e de $\mathrm{Pi}-\mathrm{NaHCO}_{3}$ extraída do solo sob SCC foi homogênea no perfil do solo, enquanto sob SPD ocorreu um forte gradiente a partir da superfície; em todas as camadas os teores desse foram superiores aos do SCC. A cultura precedente não afetou os teores destas duas frações de P (Tabela 3). Os maiores teores destas frações no SPD foram causados pelas constantes aplicações de fertilizantes fosfatados sem o posterior revolvimento do solo, e também pelo fato de o solo sob SPD ter sido mobilizado para incorporação do calcário, seis anos após a implantação do experimento.

Os teores de $\mathrm{P}$ inorgânico extraídos com $\mathrm{NaOH}$ $0,1 \mathrm{~mol} \mathrm{~L}^{-1}$ no LVd foram superiores no SPD, comparativamente aos do SCC, em todas as camadas, representando, em média, $46,6 \%$ e $47,5 \%$ do Pi total. Com o $\mathrm{NaOH} 0,5 \mathrm{~mol} \mathrm{~L}^{-1}$, recuperou-se mais Pi no SPD do que no SCC, nas duas camadas mais superficiais (Tabela 3). Isso representa 17,2\% e 21,6\% do Pi total. Os teores de P-residual não diferiram, devido aos métodos de preparo do solo, às culturas precedentes, ou às camadas amostradas.

Os fosfatos de Ca secundários extraídos do LVd, $\mathrm{Pi}-\mathrm{HCl}$, foram muito superiores no SPD do que no SCC, especialmente na camada superficial (Tabela 3). 
Este comportamento reforça a hipótese de que adições constantes de fertilizantes fosfatados na superfície do SPD aumentam muito o P da solução do solo e pode ocorrer a neoformação de fosfatos de $\mathrm{Ca}$ de menor solubilidade.

$\mathrm{O}$ teor de $\mathrm{P}$ extraído pelo método de Mehlich I no LVd foi maior no SPD do que no SCC, em todas as camadas amostradas, chegando a $146 \mathrm{mg} \mathrm{dm}^{-3}$ na camada de 0-2,5 cm (Tabela 3), o que corresponde a mais de 16 vezes o nível crítico $\left(9 \mathrm{mg} \mathrm{dm}^{-3}\right)$ (Comissão de Fertilidade do Solo, 1995). Os valores não foram afetados pelas culturas precedentes.

Deste modo, os dados do LVd mostram que o $\mathrm{P}$ do solo sob SPD distribuiu-se preferencialmente naquelas formas coordenadas ao $\mathrm{Fe}$ e ao $\mathrm{Al}$, com menor energia (resina, bicarbonato e $\mathrm{NaOH} 0,1 \mathrm{~mol} \mathrm{~L}^{-1}$ ), e nos fosfatos de Ca secundários. Também, parece que os sítios de maior avidez por $\mathrm{P}$ estão saturados, uma vez que os teores deste nutriente no extrato de $\mathrm{NaOH} 0,5 \mathrm{~mol} \mathrm{~L}^{-1}$ e no resíduo não foram afetados pelos métodos de preparo do solo e culturas precedentes.

Os teores de $\mathrm{P}$ total no solo $\mathrm{PVd}$ variaram de 468 a $759 \mathrm{mg} \mathrm{dm}^{-3}$ (Tabela 4). No SCC, os valores foram uniformes até os $7,5 \mathrm{~cm}$, com queda na camada $7,5-17,5 \mathrm{~cm}$. No SPD, ocorreu um acúmulo acentuado de $P$ total na camada $0-2,5 \mathrm{~cm}$, declinando com a profundidade. Este comportamento é típico do SPD, pois a mobilidade do $\mathrm{P}$ no solo é muito baixa.

No PVd, a quantidade de Pi-resina extraída do solo sob SCC $\left(33,26\right.$ e $\left.15 \mathrm{mg} \mathrm{dm}^{-3}\right)$ teve menor gradiente no perfil do que sob $\operatorname{SPD}(60,32 \mathrm{e}$ $15 \mathrm{mg} \mathrm{dm}^{-3}$ ), sendo superior, neste sistema, na camada superficial. Também, independentemente dos métodos de preparo do solo ou das camadas amostradas, a sucessão aveia/milho manteve os níveis de Pi-resina mais elevados, comparativamente aos da sucessão aveia+ervilhaca/milho+caupi (Tabela 4). O mesmo comportamento foi observado com o extrator bicarbonato. Somando-se o Pi-resina

Tabela 3. Fósforo total e frações de fósforo inorgânico do Latossolo Vermelho distrófico típico em razão de métodos de preparo do solo, culturas precedentes e camadas amostradas ${ }^{(1)}$.

\begin{tabular}{|c|c|c|c|c|c|c|c|}
\hline \multirow{2}{*}{$\begin{array}{l}\text { Fração de } \\
\text { fósforo }\end{array}$} & \multirow{2}{*}{$\begin{array}{c}\text { Preparo } \\
\text { do solo }\end{array}$} & \multicolumn{3}{|c|}{ Camadas amostradas $(\mathrm{cm})^{(2)}$} & \multicolumn{2}{|c|}{ Culturas precedentes $^{(3)}$} & \multirow[t]{2}{*}{ C.V. } \\
\hline & & $0-2,5$ & $2,5-7,5$ & $7,5-17,5$ & Sorgo & Soja & \\
\hline & & \multicolumn{5}{|c|}{ - } & $(\%)$ \\
\hline \multirow[t]{2}{*}{$\mathrm{P}$ total } & SPD & $1.085 \mathrm{aA}$ & $1.068 \mathrm{aA}$ & $897 \mathrm{bA}$ & & & \\
\hline & SCC & $715 \mathrm{aB}$ & $785 \mathrm{aB}$ & $749 \mathrm{aA}$ & $878 \mathrm{a}$ & $888 \mathrm{a}$ & 8,1 \\
\hline \multirow[t]{2}{*}{ Pi-resina } & SPD & $88 \mathrm{aA}$ & $69 \mathrm{bA}$ & $41 \mathrm{cA}$ & & & \\
\hline & SCC & $29 \mathrm{aB}$ & $28 \mathrm{aB}$ & $24 \mathrm{aB}$ & $47 \mathrm{a}$ & $46 \mathrm{a}$ & 27,6 \\
\hline \multirow{2}{*}{$\begin{array}{l}\mathrm{Pi}-\mathrm{NaHCO}_{3} \\
0,5 \mathrm{~mol} \mathrm{~L}^{-1}\end{array}$} & SPD & $47 \mathrm{aA}$ & $47 \mathrm{aA}$ & $28 \mathrm{bA}$ & & & \\
\hline & SCC & $20 \mathrm{aB}$ & $22 \mathrm{aB}$ & $18 \mathrm{aB}$ & $30 \mathrm{a}$ & $31 \mathrm{a}$ & 23,3 \\
\hline \multirow{2}{*}{$\begin{array}{l}\mathrm{Pi}-\mathrm{NaOH} \\
0,1 \mathrm{~mol} \mathrm{~L}^{-1}\end{array}$} & SPD & $347 \mathrm{aA}$ & $345 \mathrm{aA}$ & $287 \mathrm{bA}$ & & & \\
\hline & SCC & $216 \mathrm{aB}$ & $224 \mathrm{aB}$ & $220 \mathrm{aB}$ & $289 a$ & $257 \mathrm{~b}$ & 12,5 \\
\hline \multirow{2}{*}{$\begin{array}{l}\mathrm{Pi}-\mathrm{HCl} \\
1,0 \mathrm{~mol} \mathrm{~L}^{-1}\end{array}$} & SPD & $59 \mathrm{aA}$ & $50 \mathrm{aA}$ & $35 \mathrm{bA}$ & & & \\
\hline & SCC & $23 \mathrm{aB}$ & $26 \mathrm{aB}$ & $26 \mathrm{aB}$ & $35 a$ & $38 \mathrm{a}$ & 29,8 \\
\hline \multirow{2}{*}{$\begin{array}{l}\mathrm{Pi}-\mathrm{NaOH} \\
0,5 \mathrm{~mol} \mathrm{~L}^{-1}\end{array}$} & SPD & $122 \mathrm{aA}$ & $123 \mathrm{aA}$ & $114 \mathrm{bA}$ & & & \\
\hline & SCC & $94 \mathrm{bB}$ & $102 \mathrm{aB}$ & $102 \mathrm{aA}$ & $108 \mathrm{a}$ & $112 \mathrm{a}$ & 3,9 \\
\hline \multirow[t]{2}{*}{ P-residual } & SPD & $259 \mathrm{aA}$ & $268 \mathrm{aA}$ & $272 \mathrm{aA}$ & & & \\
\hline & SCC & $237 \mathrm{aA}$ & $262 \mathrm{aA}$ & $258 \mathrm{aA}$ & $233 a$ & $245 a$ & 8,2 \\
\hline \multirow[t]{2}{*}{ P-Mehlich } & SPD & $146 \mathrm{bA}$ & $161 \mathrm{aA}$ & $69 \mathrm{cA}$ & & & \\
\hline & SCC & $37 \mathrm{bB}$ & $52 \mathrm{aB}$ & $41 \mathrm{bB}$ & $87 a$ & $82 a$ & 17,2 \\
\hline
\end{tabular}


e o Pi-NaHCO $\mathrm{N}_{3}$, o SPD manteve muito mais $\mathrm{P}$ disponível na camada superficial do que o SCC (Tabela 4).

$\mathrm{O} \mathrm{NaOH}$ 0,1 mol L-1 no PVd também foi sensível em detectar as diferenças entre as sucessões de cultura, recuperando sempre mais P inorgânico moderadamente lábil no solo cultivado com aveia/milho. Somente na camada 7,5-17,5 cm sob SPD o teor foi menor do que o das camadas superficiais e do que o do sob SCC. Os teores de P inorgânico obtidos com a segunda extração com hidróxido foram maiores nas camadas superficiais do SPD em relação aos da camada 7,5-17,5 cm, e maior no SPD do que os do SCC na camada 0-2,5 cm (Tabela 4).

Os fosfatos de Ca de baixa solubilidade extraídos do PVd acumularam-se em grandes quantidades na camada superficial do SPD, e chegaram a valores semelhantes aos extraídos pela resina (Tabela 4). Quando da adição de fertilizantes fosfatados na forma de Ca, uma parte considerável permanece na superfície nesta fração ou é neoformado a partir dos íons fosfatos e Ca da solução do solo, correlacionando-se com o P total e disponível (resina, bicarbonato e hidróxido), até porque os sítios de adsorção devem estar praticamente saturados em face dos baixos teores de argila deste solo.

$\mathrm{O}$ teor de $\mathrm{P}$ extraído pelo método de Mehlich I no PVd foi maior na camada superficial do SPD $\left(146 \mathrm{mg} \mathrm{dm}^{-3}\right)$ do que na do SCC $\left(81 \mathrm{mg} \mathrm{dm}^{-3}\right)$, e também maior na sucessão aveia/milho $\left(85 \mathrm{mg} \mathrm{dm}^{-3}\right)$ do que na aveia+ervilhaca/milho+caupi $\left(61 \mathrm{mg} \mathrm{dm}^{-3}\right)$ (Tabela 4). Similarmente aos outros dois solos, os valores são muitas vezes superiores ao nível crítico estabelecido pela Comissão de Fertilidade do Solo (1995), que é de $14 \mathrm{mg} \mathrm{dm}^{-3}$.

Deste modo, a camada superficial do solo PVd sob SPD apresentou maiores teores de Pi-resina, $\mathrm{Pi}-\mathrm{NaHCO}_{3}, \mathrm{Pi}-\mathrm{HCl}, \mathrm{Pi}-\mathrm{NaOH}$ 0,5 e P-Mehlich I em comparação com o teor do SCC. A sucessão aveia/milho manteve maiores teores de Pi-resina, $\mathrm{Pi}-\mathrm{NaHCO}_{3}$, Pi-NaOH 0,1 e P-Mehlich I, em comparação com o de aveia + ervilhaca/milho + caupi.

Tabela 4. Fósforo total e frações de fósforo inorgânico (Pi) do Argissolo Vermelho distrófico típico em razão de métodos de preparo do solo, sucessões de cultura e camadas amostradas ${ }^{(1)}$.

\begin{tabular}{|c|c|c|c|c|c|c|c|}
\hline \multirow{2}{*}{$\begin{array}{l}\text { Fração de } \\
\text { fósforo }\end{array}$} & \multirow{2}{*}{$\begin{array}{l}\text { Preparo } \\
\text { do solo }\end{array}$} & \multicolumn{3}{|c|}{ Camadas amostradas $(\mathrm{cm})^{(2)}$} & \multicolumn{2}{|c|}{ Sucessões de cultura ${ }^{(3)}$} & \multirow[t]{2}{*}{ C.V. } \\
\hline & & $0-2,5$ & $2,5-7,5$ & $7,5-17,5$ & $\mathrm{~A} / \mathrm{M}$ & $\mathrm{A}+\mathrm{V} / \mathrm{M}+\mathrm{C}$ & \\
\hline P total & $\begin{array}{l}\text { SPD } \\
\text { SCC }\end{array}$ & $\begin{array}{l}759 \mathrm{aA} \\
537 \mathrm{aB}\end{array}$ & $\begin{array}{l}627 \mathrm{bA} \\
546 \mathrm{aB}\end{array}$ & $\begin{array}{l}-\left(\mathrm{mg} \mathrm{dm}^{-}\right. \\
468 \mathrm{cA} \\
479 \mathrm{bA}\end{array}$ & 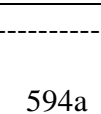 & - & $(\%)$ \\
\hline Pi-resina & $\begin{array}{l}\text { SPD } \\
\text { SCC }\end{array}$ & $\begin{array}{l}60 \mathrm{aA} \\
33 \mathrm{aB}\end{array}$ & $\begin{array}{l}32 \mathrm{bA} \\
26 \mathrm{abA}\end{array}$ & $\begin{array}{l}15 \mathrm{cA} \\
15 \mathrm{bA}\end{array}$ & $34 a$ & $26 \mathrm{~b}$ & 27,4 \\
\hline $\begin{array}{l}\mathrm{Pi}-\mathrm{NaHCO}_{3} \\
0,5 \mathrm{~mol} \mathrm{~L}^{-1}\end{array}$ & $\begin{array}{l}\text { SPD } \\
\text { SCC }\end{array}$ & $\begin{array}{l}46 \mathrm{aA} \\
28 \mathrm{aB}\end{array}$ & $\begin{array}{l}29 \mathrm{bA} \\
27 \mathrm{aA}\end{array}$ & $\begin{array}{l}14 \mathrm{cA} \\
17 \mathrm{bA}\end{array}$ & $31 \mathrm{a}$ & $23 b$ & 28,1 \\
\hline $\begin{array}{l}\mathrm{Pi}-\mathrm{NaOH} \\
0,1 \mathrm{~mol} \mathrm{~L}^{-1}\end{array}$ & $\begin{array}{l}\text { SPD } \\
\text { SCC }\end{array}$ & $\begin{array}{l}189 \mathrm{aA} \\
166 \mathrm{aA}\end{array}$ & $\begin{array}{l}164 \mathrm{aA} \\
169 \mathrm{aA}\end{array}$ & $\begin{array}{l}114 \mathrm{bB} \\
144 \mathrm{aA}\end{array}$ & $173 a$ & $142 \mathrm{~b}$ & 12,3 \\
\hline $\begin{array}{l}\mathrm{Pi}-\mathrm{HCl} \\
1,0 \mathrm{~mol} \mathrm{~L}\end{array}$ & $\begin{array}{l}\text { SPD } \\
\text { SCC }\end{array}$ & $\begin{array}{l}58 \mathrm{aA} \\
21 \mathrm{aB}\end{array}$ & $\begin{array}{l}33 \mathrm{bA} \\
22 \mathrm{aA}\end{array}$ & $\begin{array}{l}21 \mathrm{cA} \\
15 \mathrm{aA}\end{array}$ & $30 \mathrm{a}$ & $25 \mathrm{a}$ & 26,1 \\
\hline $\begin{array}{l}\mathrm{Pi}-\mathrm{NaOH} \\
0,5 \mathrm{~mol} \mathrm{~L}^{-1}\end{array}$ & $\begin{array}{l}\text { SPD } \\
\text { SCC }\end{array}$ & $\begin{array}{l}71 \mathrm{aA} \\
53 \mathrm{aB}\end{array}$ & $\begin{array}{l}60 \mathrm{abA} \\
55 \mathrm{aA}\end{array}$ & $\begin{array}{l}49 \mathrm{bA} \\
52 \mathrm{aA}\end{array}$ & $59 a$ & $54 \mathrm{a}$ & 7,4 \\
\hline P-residual & $\begin{array}{l}\text { SPD } \\
\text { SCC }\end{array}$ & $\begin{array}{l}124 \mathrm{aA} \\
106 \mathrm{aA}\end{array}$ & $\begin{array}{l}118 \mathrm{aA} \\
110 \mathrm{aA}\end{array}$ & $\begin{array}{l}127 \mathrm{aA} \\
112 \mathrm{aA}\end{array}$ & $118 \mathrm{a}$ & $114 a$ & 21,4 \\
\hline P-Mehlich & $\begin{array}{l}\text { SPD } \\
\text { SCC }\end{array}$ & $\begin{array}{r}146 \mathrm{aA} \\
81 \mathrm{aB}\end{array}$ & $\begin{array}{l}85 \mathrm{bA} \\
70 \mathrm{aA}\end{array}$ & $\begin{array}{l}28 \mathrm{cA} \\
31 \mathrm{bA}\end{array}$ & $85 a$ & $61 \mathrm{~b}$ & 26,7 \\
\hline
\end{tabular}

(1) Médias seguidas pela mesma letra, minúscula na linha e maiúscula na coluna, não diferem pelo teste de Tukey (p<0,05). ${ }^{(2)}$ Média das sucessões de cultura. ${ }^{(3)} \mathrm{A} / \mathrm{M}$ : aveia/milho; A+V/M+C: aveia+ervilhaca/milho+caupi; média dos dois métodos de preparo do solo e as três camadas amostradas. ${ }^{(4)}$ SPD: sistema plantio direto; SCC: sistema de cultivo convencional. 
A utilização de diferentes sucessões de cultura, no solo PVd cultivado exclusivamente sob SPD desde 1983, não alterou o conteúdo de $P$ total (Tabela 5), embora a produção de milho fosse maior nas sucessões aveia+ervilhaca/milho+caupi e guandu+milho, como apresentado por Bayer (1996), proporcionando maior exportação de $\mathrm{P}$ pelos grãos, em comparação com a de aveia/milho. Deste modo, a introdução de plantas como o caupi e o guandu parece ter reciclado maior quantidade de $\mathrm{P}$ de camadas abaixo de $17,5 \mathrm{~cm}$. Houve um acúmulo de $\mathrm{P}$ total na camada superficial em relação às mais profundas.

No PVd cultivado exclusivamente sob SPD, o cultivo de aveia/milho desde 1983 manteve maiores teores de P-resina do que o cultivo das sucessões aveia + ervilhaca/milho + caupi e guandu + milho Por outro lado, na sucessão guandu+milho foram recuperados maiores teores de $\mathrm{Pi}-\mathrm{NaHCO}_{3}$ em relação aos das outras duas sucessões, e menores teores de P-residual em relação a aveia/milho. De modo geral, os teores de $\mathrm{P}$ recuperado por todos os extratores diminuíram em profundidade, com exceção do P-resina no solo cultivado com guandu+milho. Os teores de P-Mehlich I também foram maiores na sucessão aveia/milho e menores na guandu+milho na camada superficial, e na camada 7,5-17,5 $\mathrm{cm}$ o teor foi maior (Tabela 5).

Os teores de $\mathrm{P}$ total de todos os solos cultivados, do presente estudo, foram maiores do que nos seus ambientes naturais (Machado et al., 1993). Isto se deve, basicamente, ao fato de as quantidades adicionadas como fertilizantes serem superiores às exportadas pelos grãos. A elevação dos teores de $\mathrm{P}$ total pode alterar o equilíbrio natural entre suas formas no solo, sua relação com os demais nutrientes, a biomassa microbiana, e as taxas de transferência entre os ambientes terrestre e aquático (Sharpley et al., 1995). A movimentação de $\mathrm{P}$ do solo nos ambientes aquáticos é favorecida pelo uso inadequado do solo,

Tabela 5. Fósforo total e frações de fósforo inorgânico (Pi) do Argissolo Vermelho distrófico típico cultivado exclusivamente sob o sistema plantio direto em razão de sucessões de cultura e camadas amostradas ${ }^{(1)}$.

\begin{tabular}{|c|c|c|c|c|c|c|c|}
\hline \multirow{2}{*}{$\begin{array}{l}\text { Fração de } \\
\text { fósforo }\end{array}$} & \multicolumn{3}{|c|}{ Sucessões de cultura ${ }^{(2)}$} & \multicolumn{3}{|c|}{ Camadas amostradas $(\mathrm{cm})^{(3)}$} & \multirow[t]{2}{*}{ C.V } \\
\hline & $\mathrm{A} / \mathrm{M}$ & $\mathrm{A}+\mathrm{V} / \mathrm{M}+\mathrm{C}$ & $\mathrm{G}+\mathrm{M}$ & $0-2,5$ & $2,5-7,5$ & $7,5-17,5$ & \\
\hline &  & ---------- & 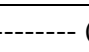 & $\left.n^{-3}\right)----$ & $\begin{array}{c}------- \\
---1\end{array}$ & ------------ & $(\%)$ \\
\hline $\mathrm{P}$ total & $657 \mathrm{a}$ & $599 \mathrm{a}$ & $662 \mathrm{a}$ & $883 a$ & $581 b$ & $454 \mathrm{c}$ & 11,5 \\
\hline $\begin{array}{l}\text { Pi-resina } \\
\text { A/M } \\
\text { A+V/M+C } \\
\text { G+M }\end{array}$ & & & & $\begin{array}{r}137 \mathrm{aA} \\
58 \mathrm{aB} \\
43 \mathrm{aB}\end{array}$ & $\begin{array}{l}67 \mathrm{bA} \\
31 \mathrm{bB} \\
30 \mathrm{aB}\end{array}$ & $\begin{array}{l}72 \mathrm{bA} \\
17 \mathrm{cC} \\
31 \mathrm{aB}\end{array}$ & 13,4 \\
\hline $\begin{array}{l}\mathrm{Pi}-\mathrm{NaHCO}_{3} \\
0,5 \mathrm{~mol} \mathrm{~L}^{-1}\end{array}$ & $20 \mathrm{~b}$ & $17 b$ & $32 \mathrm{a}$ & $34 \mathrm{a}$ & $22 b$ & $13 \mathrm{c}$ & 22,8 \\
\hline $\begin{array}{l}\mathrm{Pi}-\mathrm{NaOH} \\
0,1 \mathrm{~mol} \mathrm{~L}^{-1}\end{array}$ & $163 \mathrm{a}$ & $161 \mathrm{a}$ & $157 \mathrm{a}$ & $186 \mathrm{a}$ & $159 b$ & $135 \mathrm{c}$ & 11,2 \\
\hline $\begin{array}{l}\mathrm{Pi}-\mathrm{HCl} \\
1,0 \mathrm{~mol} \mathrm{~L}^{-1}\end{array}$ & $25 \mathrm{a}$ & $23 \mathrm{a}$ & $28 \mathrm{a}$ & $36 \mathrm{a}$ & $24 b$ & $15 \mathrm{c}$ & 30,2 \\
\hline $\begin{array}{l}\mathrm{Pi}-\mathrm{NaOH} \\
0,5 \mathrm{~mol} \mathrm{~L}^{-1}\end{array}$ & $65 \mathrm{a}$ & $65 \mathrm{a}$ & $72 \mathrm{a}$ & $76 \mathrm{a}$ & $68 \mathrm{a}$ & $57 \mathrm{~b}$ & 13,0 \\
\hline P-residual & $98 \mathrm{a}$ & $93 \mathrm{ab}$ & $87 b$ & $98 \mathrm{a}$ & $91 \mathrm{ab}$ & $88 b$ & 6,2 \\
\hline $\begin{array}{l}\text { P-Mehlich } \\
\text { A/M } \\
\mathrm{A}+\mathrm{V} / \mathrm{M}+\mathrm{C} \\
\mathrm{G}+\mathrm{M} \\
\end{array}$ & & & & $\begin{array}{r}161 \mathrm{aA} \\
122 \mathrm{aB} \\
78 \mathrm{aC} \\
\end{array}$ & $\begin{array}{l}81 \mathrm{bA} \\
78 \mathrm{bA} \\
67 \mathrm{aA} \\
\end{array}$ & $\begin{array}{l}23 \mathrm{cAB} \\
14 \mathrm{cB} \\
32 \mathrm{bA} \\
\end{array}$ & 16,9 \\
\hline
\end{tabular}

${ }^{(1)}$ Médias seguidas pela mesma letra, minúscula na linha e maiúscula na coluna, não diferem pelo teste de Tukey (p<0,05). ${ }^{(2)} \mathrm{A} / \mathrm{M}$ : aveia/milho; $\mathrm{A}+\mathrm{V} / \mathrm{M}+\mathrm{C}$ : aveia+ervilhaca/milho+caupi; G+M: guandu+milho; média das camadas amostradas. ${ }^{(3)}$ Média das sucessões de cultura. 
quer em atividades agrícolas, quer nas urbanas. No caso do SPD, os teores de P total e das formas mais solúveis da camada superficial foram muito superiores aos do SCC, podendo, dessa maneira, enriquecer a água que sai deste sistema, embora as perdas, via sedimentos, sejam diminuídas drasticamente (Sharpley et al., 1995). Isto é potencializado em solos com menores quantidades de sítios de adsorção de fosfato, como no caso do Argissolo.

O cultivo de diferentes espécies de plantas anuais teve pouco efeito na dinâmica das frações inorgânicas de P. Nos três experimentos, onde as amostras foram coletadas nas parcelas sem adição de $\mathrm{N}$, houve um grande acúmulo de $\mathrm{P}$ em formas mais disponíveis (resina, bicarbonato e hidróxido) onde se cultivaram apenas gramíneas. Assim, no caso presente, parece que o efeito não se deve ao tipo de planta, mas sim à disponibilidade de N, o qual, sendo deficiente, limita a produtividade de biomassa vegetal e a exportação dos nutrientes. Caso essa deficiência fosse eliminada e a produtividade das plantas aumentasse, provavelmente os teores recuperados pelos extratores seriam semelhantes. Também, as diferentes sucessões utilizadas nos quatro solos não afetaram os teores de fosfatos de Ca da camada superficial do SPD e nem as formas mais recalcitrantes de $\mathrm{P}$ inorgânico; exceção ao fato de que o cultivo de guandu diminuiu o P-residual no Argissolo, comparativamente à aveia e aveia+caupi, provavelmente pela ação quelante de metais proporcionada pelo ácido piscídico exsudado na rizosfera (Ae et al., 1990).

\section{Conclusões}

1. A adição de doses similares de fertilizantes fosfatados ao solo provoca maior acumulação de $\mathrm{P}$ total na camada superficial sob o sistema plantio direto do que no cultivo convencional.

2. No sistema plantio direto, recuperam-se maiores teores de $\mathrm{P}$ inorgânico lábil e não-lábil ligado ao Ca na camada superficial, em comparação com o do cultivo convencional.

3. As maiores concentrações de P inorgânico encontram-se em formas moderadamente lábeis, independentemente do tipo de solo, do método de preparo, da sucessão de cultura e da camada amostrada.
4. As sucessões de cultura têm pouca influência na distribuição das frações de P inorgânico.

\section{Agradecimentos}

Ao professor João Mielniczuk (UFRGS) e aos pesquisadores Rainoldo Alberto Kochhann (Embrapa-Centro Nacional de Pesquisa de Trigo), Armando Dalla Rosa e João Becker (COTRISA), pela permissão de uso das áreas experimentais, as quais serviram de base para este trabalho.

\section{Referências}

AE, N.; ARIHARA, J.; OKADA, K.; YOSHIHARA, T.; JOHANSEN, C. Phosphorus uptake by pigeon pea and its role in cropping systems of the Indian subcontinent. Science, Washington, v. 248, p. 477-480, 1990.

ARAÚJO, M. S. B.; SALCEDO, I. H. Formas preferenciais de acumulação de fósforo em solos cultivados com cana-de-açúcar na região do Nordeste. Revista Brasileira de Ciência do Solo, Campinas, v. 21, n. 4, p. 643-650, 1997.

BALL-COELHO, B.; SALCEDO, I. H.; TIESSEN, H.; STEWART, W. B. Short and long-term phosphorus dynamics in a fertilized ultisol under sugarcane. Soil Science Society of America Journal, Madison, v. 57, n. 4, p. 1027-1034, 1993.

BAYER, C. Dinâmica da matéria orgânica em sistemas de manejo de solos. Porto Alegre : UFRGS, 1996. 240 p. Tese de Doutorado.

BECK, M. A.; SANCHEZ, P. A. Soil phosphorus fraction dynamics during 18 years of cultivation on a Typic Paleudult. Soil Science Society of America Journal, Madison, v. 58, n. 5, p. 1424-1431, 1994.

BROOKES, P. C.; POWLSON, D. C. Preventing phosphorus losses during perchloric acid digestion of sodium bicarbonate soil extracts. Journal of the Science of Food and Agriculture, Chichester, v. 32, p. 671-674, 1982.

COMISSÃO DE FERTILIDADE DO SOLO-RS/SC (Passo Fundo, RS). Recomendação de adubação e calagem para os Estados do Rio Grande do Sul e Santa Catarina. 3. ed. Passo Fundo : Sociedade Brasileira de Ciência do Solo-Núcleo Regional Sul/Embrapa-CNPT, 1995. 224 p.

CROSS, A. F.; SCHLESINGER, W. H. A literature review and evaluation of the Hedley fractionation: applications 
to the biogeochemical cycle of soil phosphorus in natural ecosystems. Geoderma, Amsterdam, v. 64, n. 3/4, p. 197-214, 1995.

DICK, W. A.; TABATABAI, M. A. Determination of orthophosphate in aqueous solutions containing labile organic and inorganic phosphorus compounds. Journal of Environmental Quality, Madison, v. 6, n. 1, p. 82-85, 1977.

GUO, F.; YOST, R. S. Partitioning soil phosphorus into three discrete pools of differing availability. Soil Science, Baltimore, v. 163, n. 10, p. 822-833, 1998.

HEDLEY, M. J.; STEWART, J. W. B.; CHAUHAN, B. S. Changes in inorganic and organic soil phosphorus fractions induced by cultivation practices and by laboratory incubations. Soil Science Society of America Journal, Madison, v. 46, n. 4, p. 970-976, 1982.

KINGERY, W. L.; WOOD, C. W.; WILLIAMS, J. C. Tillage and amendment effects on soil carbon and nitrogen mineralization and phosphorus release. Soil and Tillage Research, Amsterdam, v. 37, n. 4, p. 239-250, 1996.

KUNISHI, H. M.; BANDEL, V. A.; MULFORD, F. R. Measurement of available soil phosphorus under conventional and no-till management. Communications in Soil Science and Plant Analysis, New York, v. 13, n. 8, p. 607-618, 1982.

LAJTHA, K.; HARRISON, A. F. Strategies of phosphorus acquisition and conservation by plants species and communities. In: TIESSEN, H. (Ed.). Phosphorus in the global environment: transfers, cycles and management. Chichester : J. Wiley, 1995. p. 139-146.

MACHADO, M. I. C. S.; BRAUNER, J. L.; VIANNA, A. C. T. Formas de fósforo na camada arável de solos do
Rio Grande do Sul. Revista Brasileira de Ciência do Solo, Campinas, v. 17, n. 3, p. 331-336, 1993.

MAGID, J. Vegetation effects on phosphorus fraction in set-aside soils. Plant and Soil, Dordrecht, v. 149, n. 1, p. 111-119, 1993.

MEHLICH, A. Determination of P, Ca, Mg, K, Na and $\mathbf{N H}_{4}$. Raleigh : North Carolina Soil Testing Division, 1953. 23 p. (Publication, 1).

RHEINHEIMER, D. S.; KAMINSKI, J.; LUPATINI, G. C.; SANTOS, E. J. S. Modificações em atributos químicos de solo arenoso sob sistema plantio direto. Revista Brasileira de Ciência do Solo, Campinas, v. 22, n. 4, p. 713-721, 1998.

SCHMIDT, J. P.; BUOL, S. W.; KAMPRATH, E. J. Soil phosphorus dynamics during seventeen years of continuous cultivation: fractionation analyses. Soil Science Society of America Journal, Madison, v. 60, n. 4, p. 1168-1172, 1996.

SELLES, F.; KOCHHANN, R. A.; DENARDIN, J. E.; ZENTNER, R. P.; FAGANELLO, A. Distribution of phosphorus fractions in Brazilian oxisol under different tillage systems. Soil and Tillage Research, Amsterdam, v. 44, n. 1, p. 23-34, 1997.

SHARPLEY, A. N.; HEDLEY, M. J.; SIBBESEN, E.; HILLBRICHT-ILKOWSKA, A.; HOUSE, W. A.; RYSZKOWSKI, L. Phosphorus transfers from terrestrial to aquatic ecosystems. In: TIESSEN, H. (Ed.). Phosphorus in the global environment: transfers, cycles and management. Chichester : J. Wiley, 1995. p. 171-200.

SMECK, N. E. Phosphorus dynamics in soils and landscapes. Geoderma, Amsterdam, v. 36, n. 3/4, p. 185-199, 1985.

WALKER, T. W.; SYERS, J. K. The fate of phosphorus during pedogenesis. Geoderma, Amsterdam, v. 15, n. 1, p. 1-19, 1976. 